setting, our results confirm that defSSc might represent an intermediate entity between pre-clinical stages and the most severe subsets of disease, thereby opening new perspectives on SSc pathophysiology and disease interception.

[1] Cossu M, et al. Rheumatology 2016

[2] LeRoy EC, et al. J Rheumatol 2001.

[3] van den Hoogen F, et al. Arthritis Rheum 2013.

Acknowledgements: Supported by a grant from Gruppo Italiano per la Lotta alla Sclerodermia (GILS). MC and TR are partly supported by the VIDI laureate and Dutch Arthritis Foundation (NWO, Netherlands Institute for Science) and ERC starting grant (EU) obtained by TR. The LTI MultiPlex Core Facility is acknowledged for technical performance of the multiplex immune assays.

Disclosure of Interest: None declared

DOI: 10.1136/annrheumdis-2017-eular.5295

\section{AB0178 PHENOTYPING OF NATURAL KILLER (NK) RECEPTORS ON NK AND NKT-LIKE CELLS DISCLOSES DEFECTIVE IMMUNE-REGULATORY CAPABILITY IN PATIENTS WITH SYSTEMIC SCLEROSIS}

M. Cossu $^{1,2}$, L. Beretta ${ }^{3}$, E. Trombetta ${ }^{4}$, S.T.A. van Bijnen ${ }^{5,6}$, M. Rossato ${ }^{1,2}$, L. van Bon ${ }^{1}$, M. van der Kroef ${ }^{1,2}$, E.C. Chouri ${ }^{1,2}$, C.G.K. Wichers ${ }^{1,2}$,

N. Vazirpanah ${ }^{1,2}$, M.C. Vonk ${ }^{7}$, L. Porretti ${ }^{4}$, F. Preijers ${ }^{6}$, H. Dolstra ${ }^{6}$,

T.R.D.J. Radstake ${ }^{1,2} .{ }^{1}$ Rheumatology \& Clinical Immunology; ${ }^{2}$ Laboratory of Translational Immunology (LTI), UMC Utrecht, Utrecht, Netherlands; ${ }^{3}$ Referral Center for Systemic Autoimmune Diseases; ${ }^{4}$ Flow Cytometry Service, Clinical Chemistry and Microbiology Laboratory, Fondazione IRCCS Ca' Granda Ospedale Maggiore Policlinico, Milano, Italy; ${ }^{5}$ Hematology; ${ }^{6}$ Laboratory Medicine, Laboratory of Hematology; ${ }^{7}$ Rheumatology, Radboud University Medical Center, Nijmegen, Netherlands

Background: Systemic sclerosis (SSc) is an autoimmune disease characterized by dysregulation of the immune system, vasculopathy and fibrosis of the skin and internal organs. Natural Killer $\left(C D 56^{+}\right.$CD3-, NK) and NKT-like $\left(C D 56^{+} \mathrm{CD}^{+}\right)$cells display receptors (NKR) whose expression pattern determines their cytotoxic and immune-regulatory activity. The role of NK and NKT-like cells in the dysregulation of the immune system in SSc has not been fully elucidated yet.

Objectives: To improve our knowledge on the contribution of NK, NKT-like and a subset of NKT cells expressing invariant TCR (iNKT) in SSc development, we performed a broad phenotyping of NKR in the circulation of SSc patients, including subjects with pre-clinical SSc.

Methods: NKR were assessed by flow cytometry using two 13-color panels on whole blood of 84 SSc patients and 20 healthy controls (HC). In particular, 15 patients with early SSc (EaSSc) without signs or symptoms of evolutive disease (2001 LeRoy and Medsger criteria ${ }^{1}$ ), 24 patients with definite SSc without skin or lung fibrosis (defSSc), 26 patients with limited (IcSSc) and 19 patients with diffuse cutaneous SSc (dcSSc) (2013 ACR/EULAR classification criteria for SSc $^{2}$ ) were included. NK degranulation in response to K562 target cells was assessed in IcSSc and dcSSc patients versus HC

Results: The number of circulating lymphocytes, NKT-like and iNKT cells - but neither $\mathrm{CD}^{+} \mathrm{T}$ nor NK cells - was reduced in dcSSc versus HC. NKp46+ NK cells co-expressing NKG2D and CD16 were decreased in dcSSc versus $\mathrm{HC}$ and EaSSc. Consistently with these observations, dcSSc exhibited lower degranulation capability. $\left(\mathrm{CD} 57^{+}\right) \mathrm{KIR}^{+}$and activating NKR-expressing NKT-like cells were diminished in both dcSSc and IcSSc versus $\mathrm{HC}$.

Conclusions: dcSSc patients showed a defective NK cytotoxicity potential, possibly due to the decreased $\mathrm{NKp}_{4} 6^{+}$fraction. The regulatory, cytolytic $\mathrm{KIR}^{+}$ NKT-like fraction was also reduced with a parallel decrease of activating receptors expression in both IcSSc and dcSSc. Overall these results point towards an impairment of NK and NKT-like cells as immune check-points in fibrotic SSc.

References:

[1] LeRoy EC, et al. J Rheumatol 2001.

[2] van den Hoogen F, et al. Arthritis Rheum 2013

Acknowledgements: Supported by a grant from Gruppo Italiano per la Lotta alla Sclerodermia (GILS). MC and TR are partly supported by the VIDI laureate and Dutch Arthritis Foundation (NWO, Netherlands Institute for Science) and ERC starting grant (EU) obtained by TR. The authors would like to thank Koos Gaiser (U-DAIR, LTI, UMC Utrecht) for the technical assistance in developing the 13-color flow cytometry panels; Dr F. Montero, L. Nieto-Gligorovski and E. Gautherot (Beckman Coulter Inc, Marseille) for providing the NKG2A-PB antibody; the NIH Tetramer Facility for providing the CD1d tetramers.

Disclosure of Interest: None declared

DOI: 10.1136/annrheumdis-2017-eular.5059

\section{AB0179 DEGRADATION OF TYPE VII COLLAGEN (C7M) IS ASSOCIATED WITH SYSTEMIC SCLEROSIS - DEVELOPMENT OF A NOVEL NEO-EPITOPE SPECIFIC ASSAY}

J. Sand ${ }^{1}$, P. Juhl ${ }^{1}$, L. Iversen ${ }^{2}$, T. Karlsmark ${ }^{2}$, M. Karsdal ${ }^{1}$, A.-C. Bay-Jensen ${ }^{1}$, M. Mogensen ${ }^{2}$, A.S. Siebuhr ${ }^{1}$, D. Leeming ${ }^{1}$. ${ }^{1}$ Nordic Bioscience, Herlev;

${ }^{2}$ Department of dermatology, Bispebjerg Hospital, Copenhagen, Denmark

Background: Type VII collagen (col7) is the main component of the anchoring fibrils that connects the basement membrane to the underlying interstitial matrix and has mainly been investigated for its role in blistering skin diseases. It has been investigated for its role in dystrophic epidermolysis bullosa, a severe skin disease. Furthermore, increased levels of type VII collagen in skin has been reported for patients with systemic sclerosis (SSc).

Objectives: The objective was to develop and characterize a blood-based marker assessing col7 degradation in patients with SSc.

Methods: We identified a specific fragment of col7 in serum from COPD patients, which was not found in controls, using mass spectrometry. A monoclonal antibody was raised against the first ten amino acids of the neo-epitope (KLH-CGGGPPGPPGRLV) and employed in a competitive ELISA (C7M). The C7M assay was validated technically and was subsequently evaluated in 2 cohorts including SSc patients. The first cohort (SSc\#1; $n=35)$ consisted of early $(<2$ years of SSc symptoms; $n=16)$ and late ( $>10$ years of disease with stable skin for at least 6 months, $n=19$ ) diffuse SSc patients, while the second cohort (SSc\#2; $n=119)$ consisted of limited $(n=78)$ and diffuse $(n=41)$ SSc patients. Serum C7M levels were likewise measured in healthy subjects and compared to the levels of SSc patients using the Kruskal-Wallis test with Dunn's multiple comparisons test comparing healthy individuals with the two SSc cohorts.

Results: A technically robust competitive ELISA (C7M), which was highly specific for a col7 fragment was developed. The assay showed acceptable inter- $(13 \%)$ and intra-assay ( $9 \%)$ variation, linearity (102\% dilution recovery), analyte stability (102\% recovery after 4 freeze/thaw cycles), and interference.

The C7M marker was evaluated by comparing serum levels in healthy donors with patients with SSc (Figure). Serum C7M levels were not associated with age, gender, BMI, or disease duration. The geometric mean serum C7M level in healthy donors was $4.6 \mathrm{ng} / \mathrm{mL}(95 \% \mathrm{Cl} 3.7-5.6 \mathrm{ng} / \mathrm{mL})$. The geometric mean serum C7M levels were significantly elevated in both cohorts of patients with SSc (SSc\#1, $13.6 \mathrm{ng} / \mathrm{mL}[95 \% \mathrm{Cl} 11.1-16.5], \mathrm{p}<0.0001 ;$ SSc\#2, $9.2 \mathrm{ng} / \mathrm{mL}[95 \%$ $\mathrm{Cl}$ 8.3-10.2], $\mathrm{p}<0.0001)$. Furthermore, a significant difference were observed between the two cohorts $(P=0.05)$.

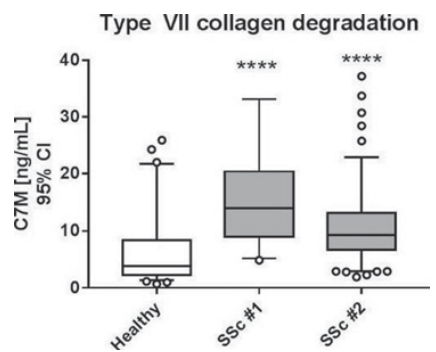

Conclusions: The C7M ELISA enabled quantification of type VII collagen degradation in serum. Elevated serum C7M levels indicated that the remodeling of type VII collagen was significantly increased in patients with SSc, suggesting a pathological role.

Acknowledgements: We thank Biogen Idec's SSc department for their contribution.

Disclosure of Interest: None declared

DOI: 10.1136/annrheumdis-2017-eular.5689

\section{AB0180 BIOMARKERS OF EXTRACELLULAR MATRIX REMODELING ARE ASSOCIATED WITH ACUTE EXACERBATIONS OF IDIOPATHIC INTERSTITIAL PNEUMONIA}

J. Sand ${ }^{1}$, P. Juhl ${ }^{1}$, Y. Tanino ${ }^{2}$, T. Nikaido ${ }^{2}$, K. Misa ${ }^{2}$, Y. Sato ${ }^{2}$, R. Togawa ${ }^{2}$ X. Wang ${ }^{2}$, M. Karsdal ${ }^{1}$, A.-C. Bay-Jensen ${ }^{1}$, D. Leeming ${ }^{1}$, A.S. Siebuhr ${ }^{1}$, M. Munakata ${ }^{2} .{ }^{1}$ Nordic Bioscience, Herlev, Denmark; ${ }^{2}$ Department of Pulmonary Medicine, Fukushima Medical University School of Medicine, Fukushima, Japan

Background: Idiopathic interstitial pneumonia (IIP) is characterized by an increased rate of extracellular matrix (ECM) turnover resulting in fibrosis. The pathogenic remodeling includes increased levels of protein synthesis and degradation mediated by proteases such as matrix metalloproteinases (MMPs). Acute exacerbations of IIP (AE-IIP) represent periods of increased disease activity. Pulmonary involvement, especially pulmonary fibrosis, is common in patients suffering from systemic sclerosis (SSc) and ankylosing spondylitis (AS)

Objectives: The objective was to investigate if ECM remodeling was altered during AE-IIP by serological neo-epitope biomarkers.

Methods: Serum samples were collected from patients with IIP at clinically stable disease (S-IIP, $n=29)$ and at AE-IIP $(n=68)$. Of these, 11 and 28 patients, respectively, had idiopathic pulmonary fibrosis (IPF). 28 IIP patients had paired samples. Biomarkers released from MMP-mediated degradation of collagen type I (C1M), III (C3M), IV (C4M), and VI (C6M), elastin (ELM7), versican (VCANM), biglycan (BGM), and C-reactive protein (CRPM) were assessed in serum by competitive ELISAs utilizing neo-epitope specific monoclonal antibodies. Data were analysed using Mann-Whitney test, Wilcoxon test, Spearman's rank correlation, and Kaplan-Meier curves as appropriate.

Results: Mean age of patients was 71 (range 54-86) at AE-IIP and 69 (range 55-83) at S-IIP. Mean forced vital capacity in percentage of predicted value (\%FVC) was $55.6 \%($ SD 19.5) at AE-IIP and $79.0 \%$ (SD 26.5) at S-IIP. Serum 
levels of C4M, C6M, and CRPM at AE-IIP were positively correlated with \%FVC $(r=0.37, p=0.008 ; r=0.36, p=0.011 ; r=0.33, p=0.020$; respectively). Serum levels of C4M $(p=0.002)$ and C6M $(p=0.024)$ were increased while ELM7 $(p=0.024)$ and VCANM $(p<0.0001)$ were decreased at AE-IIP as compared with $S$-IIP when analyzing all patients. IPF patients had lower levels of VCANM at AE-IPF $(p=0.0001)$. Patients with paired samples showed significantly elevated serum levels of C4M ( $p=0.004)$ and decreased levels of ELM7 and VCANM ( $p=0.036$ and $p=0.0001$, respectively) at AE-IIP. C1M and C6M levels at AE-IIP were borderline related to mortality outcome for IPF patients (both $p=0.059$ ) with levels above median associated with a higher risk of mortality. Analyses of all or paired patients showed no associations with mortality.

Conclusions: Serological levels of neo-epitope biomarkers of ECM degradation were associated with AE-IIP and weakly with mortality outcome. These results indicate that the rate of ECM remodeling in the lungs of patients with IIP is significantly altered during periods of high disease activity such as an acute exacerbation. The difference in degradation profile for the proteins studied is intriguing and indicate activation of different processes contributing to AE-IIP. Neo-epitope biomarkers of the ECM might be useful in identifying patients diagnosed with rheumatic disease as SSc and AS with pulmonary involvement. Acknowledgements: The Danish Research Foundation

Disclosure of Interest: None declared

DOI: 10.1136/annrheumdis-2017-eular.5699

\section{AB0181 DIFFUSING CAPACITY AND CLINICAL CHARACTERISTICS OF PATIENTS WITH SYSTEMIC SCLEROSIS - DATA FROM THE GERMAN NETWORK FOR SYSTEMIC SCLEROSIS}

P. Moinzadeh ${ }^{1}$, N. Blank ${ }^{2}$, E. Siegert ${ }^{3}$, J. Henes ${ }^{4}$, M. Worm ${ }^{5}$,

C. Sunderkoetter ${ }^{6}$, M. Schmalzing ${ }^{7}$, A. Kreuter ${ }^{8}$, C. Gunther $^{9}$, L. Susok ${ }^{10}$, G. Zeidler ${ }^{11}$, I. Koetter ${ }^{12}$, U. Mueller-Ladner ${ }^{13}$, T. Krieg $^{1}$, A. Juche ${ }^{14}$,

T. Schmeiser ${ }^{15}$, G. Riemekasten ${ }^{16}$, E. Aberer ${ }^{17}$, N. Gaebelein-Wissing ${ }^{18}$, J.H.W. Distler ${ }^{19}$, M. Sárdy ${ }^{20}$, C. Pfeiffer ${ }^{21}$, K. Kuhr ${ }^{22}$, N. Hunzelmann ${ }^{1}$,

F. Bonella ${ }^{23}$, M. Kreuter ${ }^{24}$. $^{1}$ Dermatology, University Hospital Cologne, Cologne;

${ }^{2}$ Rheumatology, University Hospital Heidelberg, Heidelberg; ${ }^{3}$ Rheumatology, Charité Universitätsmedizin Berlin, Berlin; ${ }^{4}$ Rheumatology, University Hospital Tuebingen, Tuebingen; ${ }^{5}$ Dermatology, Charité Universitätsmedizin Berlin, Berlin ${ }^{6}$ Dermatology, University Hospital Muenster, Muenster; ${ }^{7}$ Rheumatology, University Hospital Wuerzburg, Wuerzburg; ${ }^{8}$ Dermatology, HELIOS St. Elisabeth Klinik Oberhausen, Oberhausen; ${ }^{9}$ Dermatology, University Hospital Carl Gustav Carus, Dresden; ${ }^{10}$ Dermatology, St. Josef Hospital Bochum, Bochum; ${ }^{11}$ Rheumatology, Johanniter-Krankenhaus im Fläming Treuenbrietzen, Treuenbrietzen; ${ }^{12}$ Rheumatology, Asklepios Klinik Altona, Hamburg:

${ }^{13}$ Rheumatology, Justus Liebig University Giessen, Kerckhoff Clinic, Bad Nauheim; ${ }^{14}$ Immanuel Krankenhaus Berlin-Buch, Berlin; ${ }^{15}$ Rheumatology, Krankenhaus St. Josef, Wuppertal: ${ }^{16}$ Rheumatology, University Medical Center-UKSH, Luebeck, Germany; ${ }^{17}$ Dermatology, Medical University of Graz, Graz, Austria; ${ }^{18}$ Dermatology, HELIOS University Hospital Wuppertal, Wuppertal; ${ }^{19}$ Rheumatology, University Hospital Erlangen, Erlangen; ${ }^{20}$ Dermatology, Ludwig Maximilians University Hospital, Munich; ${ }^{21}$ Dermatology, University Medical Center UIm, UIm; ${ }^{22}$ IMSIE, University of Cologne, Cologne; ${ }^{23}$ Unit for interstitial lung diseases, Ruhrlandklinik University Hospital Essen, Essen; ${ }^{24}$ Center for interstitial and rare lung diseases, Thoraxklinik, University Hospital Heidelberg, Heidelberg, Germany

Background: Lung involvement, i.e. interstitial lung disease (ILD) and pulmonary hypertension $(\mathrm{PH})$, is common in patients with systemic sclerosis ( $\mathrm{SSc}$ ), significantly limiting quality of life and survival. Data on clinical correlations between lung function and clinical subsets of SSc are sparse.

Objectives: To investigate the relationship of DLCO and clinical characteristics in patients SSc patients within the registry of the German Network for Systemic Scleroderma.

Methods: Clinical data of the patient registry, currently including DLCO data of 1917 patients were evaluated. In total, these patients were clinically evaluated 5997 times (i.e., at the first visit and during follow-up visits). At the initial visit and during follow-up DLCO levels were correlated with clinical characteristics.

Results: At initial presentation, $64 \%$ of the patients had DLCO levels $<75 \%$ predicted. Impaired DLCO levels were observed in $74 \%$ of dcSSc patients, in $64 \%$ of SSc-Overlap patients and $57 \%$ of IcSSc patients $(p<0.0001)$. Furthermore, male patients $(62 \%)$, patients with $\mathrm{PH}(80 \%)$, ILD $(80 \%)$, dyspnea $(78 \%)$, and those with presence of anti-topoisomerase I antibodies $(71 \%)$ exhibited significantly more often DLCO levels $<75 \%(p<0.01)$. Patients suffering from dcSSc had the lowest DLCO levels (mean value, $62 \%$ ), followed by patients with SSc-Overlap syndromes (mean value, 67\%) and IcSSc patients (mean value, $70 \%$ ) revealing significant differences between subsets. Long-term follow-up evaluation (mean follow up, 6.0 years) revealed that in comparison to IcSSc patients dcSSc patients (OR 2.1; $p<0.0001 ; 95 \%>C l$ 1.7-2.5) and SSc-Overlap patients (OR, 1.55; $\mathrm{p}<0.0001 ; 95 \% \mathrm{Cl}, 1.2-2.0)$ had a significantly increased risk to a decrease in DLCO levels $<75 \%$.

Conclusions: Impairment of pulmonary function as determined by diffusing capacity DLCO is more common and more pronounced in patients with dcSSc and SSc-Overlap Syndrome compared to IcSSc. DLCO may be useful for diagnosing and monitoring pulmonary involvement in SSc.

Disclosure of Interest: None declared
DOI: 10.1136/annrheumdis-2017-eular.3474

\section{AB0182 IN VITRO CHARACTERIZATION OF DERMAL FIBROBLASTS FROM SYSTEMIC SCLEROSIS PATIENTS}

R. Brizzolara $^{1}$, P. Montagna ${ }^{1}$, S. Soldano ${ }^{1}$, A.C. Trombetta ${ }^{1}$, B. Ruaro ${ }^{1}$, A. Sulli ${ }^{1}$, S. Scabini ${ }^{2}$, E. Stratta ${ }^{2}$, V. Smith ${ }^{3}$, M. Cutolo ${ }^{4} .{ }^{1}$ Research Laboratory and Academic Division of Clinical Rheumatology, Department of Internal Medicine, University of Genoa, IRCCS San Martino; ${ }^{2}$ Oncologic Surgery, Department of Surgery, IRCCS San Martino IST, Genoa, Italy; ${ }^{3}$ Department of Rheumatology, Ghent University Hospital, Ghent, Belgium; ${ }^{4}$ Research Laboratory and Academic Division of Clinical Rheumatology, Department of Internal Medicine, University of Genoa, Genoa, Italy

Background: Systemic sclerosis (SSc) is characterized by progressive fibrosis of the skin and/or internal organs. Skin fibrosis is mainly due to the excessive production of extracellular matrix (ECM) proteins from dermal activated fibroblasts. The high amounts of transforming growth factor-beta (TGFbeta) production induce fibroblast proliferation and their transition into profibrotic myofibroblasts. Consequently, the increased presence of alpha-smooth muscle actin (alphaSMA)-positive myofibroblasts in affected tissues is associated with fibrotic tissue remodelling, that characterize SSc [1-4].

Objectives: To characterize dermal fibroblasts from SSc patients by evaluating the gene expression of specific phenotypic and profibrotic markers: fibrillar collagen type I (COL I), fibronectin (FN), fibroblast-specific protein 1 (S100A4), TGFbeta and alpha-SMA (marker of myofibroblasts).

Methods: Human dermal fibroblasts were obtained by skin biopsy from affected areas of 6 active IcSSc patients after written informed consent and approval of medical ethics committee. SSc patients fulfilled the new EULAR/ACR criteria for SSc and they were treated only with various vasodilators (no severe clinical SSc complications were present at the time of skin sampling). Human SSc dermal fibroblasts were cultured up to $80 \%$ of confluence and at the third to fifth subpassages were used for experiments. Total RNA was extracted and quantitative real-time PCR (qRT-PCR) was performed to evaluate the gene expression of relevant fibroblast markers: COL I, FN, S100A4, TGFbeta and alpha-SMA.

Results: In human SSc fibroblasts the gene expression of the relevant markers $\mathrm{COL} \mathrm{I,} \mathrm{FN} \mathrm{and} \mathrm{S100A4} \mathrm{resulted} \mathrm{of} 10^{7}$ RNA copies for each gene. Interestingly, in the same tested fibroblasts, the profibrotic alpha-SMA gene expression (marker of myofibroblasts) was similarly found highly expressed, with a result of $10^{7}$ gene copies. On the contrary, TGFbeta showed a much lower gene expression $\left(10^{3}\right.$ copies) compared to the other investigated genes.

Conclusions: In human SSc cultured dermal fibroblasts, the gene expression of ECM proteins ( $\mathrm{COL} I$ and $\mathrm{FN}$ ), was found associated with a relevant gene expression of S100A4 and alpha-SMA, confirming their myofibroblast phenotype, as highly differentiated. Interestingly, TGFbeta gene appears to be less expressed. It could be concluded that myofibroblasts from the SSc skin mainly undergo the effects of the tissutal TGFbeta, since it seems that they do not contribute to its further production once differentiated.

References:

[1] Cutolo M. et al. J Rheumatol 2015;42:3.

[2] Brunasso A.M. et al. F1000Research 2016;5:723.

[3] Hinz B et al. Am J Pathol 2012;180:1340-55.

[4] Dumoitier N. et al. Arthritis Rheumatol 2016 [Epub ahead of print].

Disclosure of Interest: None declared

DOI: 10.1136/annrheumdis-2017-eular.3687

\section{AB0183 ISOLATION AND CHARACTERIZATION OF CIRCULATING FIBROCYTES FROM SYSTEMIC SCLEROSIS PATIENTS: AN VITRO INVESTIGATION}

R. Brizzolara ${ }^{1}$, P. Montagna ${ }^{1}$, S. Soldano ${ }^{1}$, A.C. Trombetta $^{1}$, P. Contini ${ }^{2}$ V. Smith ${ }^{3}$, B. Ruaro ${ }^{1}$, A. Sulli ${ }^{1}$, M. Cutolo ${ }^{1}$. ${ }^{1}$ Research Laboratory and Academic Division of Clinical Rheumatology, Department of Internal Medicine, University of Genoa, IRCCS San Martino; ${ }^{2}$ Division of Clinical Immunology, Department of Internal Medicine, University of Genoa, Genoa, Italy; ${ }^{3}$ Department of Rheumatology, Ghent University Hospital, Ghent University, Ghent, Belgium

Background: In physiological and pathological conditions, such as in tissue repair and in fibrosing diseases (i.e. systemic sclerosis, SSc), the importance of circulating fibrocytes relies on the capacity of such cells to migrate into scleroderma-affected tissues (through CXCR4/CXCL12 interaction) and to differentiate into fibroblasts/myofibroblasts, inducing fibrosis (through matrix protein deposition) (or both) [1-3]. In addition, fibrocytes seem to exert immunomodulatory effects, expressing class II major histocompatability complex molecules (HLA-DP, -DQ, and -DR) [4].

Objectives: To isolate fibrocytes from Circulating Progenitor Cells (CPCs peripheral blood mononuclear cells PBMCs) of SSc patients and healthy subjects and to identify them by fluorescence-activated cell sorter analysis (FACS), using their specific markers: the leukocyte common antigen CD45, collagen I (COL I), the chemokine receptor CXCR4 and HLA-DR [2]

Methods: Samples were collected, at basal time (t0), from 11 SSc patients, affected by SSc (treated only with different vasodilator drugs) and 5 healthy 